Review Article

\title{
Potential Acupoint Prescriptions and Outcome Reporting for Acupuncture in Atopic Eczema: A Scoping Review
}

\author{
Zhiwen Zeng ${ }^{(D)},{ }^{1,2}$ Man Li $\left(\mathbb{D},{ }^{2}\right.$ Yunjie Zeng ${ }^{D},{ }^{2}$ Jialing Zhang $\left(\mathbb{D},{ }^{2}\right.$ Yingjie Zhao $\mathbb{D}^{2},{ }^{2}$ \\ Yuanxun Lin $\mathbb{D}{ }^{2}$, Ruijin Qiu $\mathbb{D}^{3},{ }^{3}$ Dong-Shu Zhang $\mathbb{D}^{1,2}$ and Hong-Cai Shang $\mathbb{D}^{3}$ \\ ${ }^{1}$ Nanfang Hospital, Southern Medical University, Guangzhou, Guangdong, China \\ ${ }^{2}$ School of Traditional Chinese Medicine, Southern Medical University, Guangzhou, Guangdong, China \\ ${ }^{3}$ Dongzhimen Hospital of Beijing University of Chinese Medicine, Beijing, China
}

Correspondence should be addressed to Ruijin Qiu; qiuruijin@bucm.edu.cn, Dong-Shu Zhang; nyzds@sina.com, and HongCai Shang; shanghongcai@foxmail.com

Received 11 March 2021; Accepted 17 June 2021; Published 28 June 2021

Academic Editor: Chan-Yen Kuo

Copyright (C) 2021 Zhiwen Zeng et al. This is an open access article distributed under the Creative Commons Attribution License, which permits unrestricted use, distribution, and reproduction in any medium, provided the original work is properly cited.

\begin{abstract}
Background. Acupuncture is considered a complementary therapy for atopic eczema. The aim of this scoping review is to identify, examine, and summarize the potential acupoint prescriptions and outcome reporting regarding the clinical trials of acupuncture for eczema. Methods. We searched different databases from inception to September 30, 2020. The data were screened and extracted to identify the potential acupuncture prescription and examine the variation in outcome reporting, outcome measurement instruments (OMIs), and measurement time points in clinical trials of acupuncture. Results. A total of 116 clinical studies were included. The acupoint combination of LI11 and SP10 was used frequently. The core acupoint association networks were acupoints LI11, SP10, ST36, SP6, and LI4. For clinical trials of acupuncture, a total of 6 outcome distinct domains were identified in the 32 outcome measurements. The most frequently reported outcome was the eczema area, which was reported 97 times $(83.6 \%, 97 / 116)$. Immune system outcomes were assessed in 15 outcome measurements, which totally reported 37 times. Adverse events were reported 51 times. TCM syndrome, which could reflect the characteristics of TCM, was reported 4 times. 29 outcomes $(90.6 \%, 29 / 32)$ were provided definitions or OMIs. Among these outcomes, the outcome measurement times ranged from 0 to 34 . Conclusions. This scoping review provides potential knowledge that should be considered as priority in future research of acupuncture for eczema.
\end{abstract}

\section{Introduction}

Atopic eczema (AE) is a chronic inflammatory skin disease, clinically characterized by exacerbations and remissions of eczematous skin with inflammation, pruritus and excoriations, scaling, dry skin, and susceptibility for cutaneous bacterial and mycotic infections [1]. In the past 30 years, lifetime prevalence has shown a worldwide increase, which plateaus at $10-20 \%$ in developed countries and continues to increase in China and many developing countries but lower [2]

The concurrent use of terms such as "eczema," "atopic eczema," "atopic dermatitis," "atopic eczema/dermatitis syndrome," and "neurodermatitis" has led to confusion and inconsistency in their application. In China, AE is also called as chronic eczema (CE) [3]. Therefore, the nomenclature review committee of the World Allergy Organization has proposed the use of "eczema" as a unifying term, and this term is also used throughout the text of this review [4].

Acupuncture was frequently applied in cases of allergy [5]. In recent years, it has been increasingly utilized as an adjuvant therapy to conventional treatment of eczema [6]. A large number of clinical trials related to acupuncture for eczema have been published in various journals [7]. However, evidence of acupuncture for eczema keeps unclear. The treatment prescriptions are various, and outcome reporting showing heterogeneity makes it impossible to merge data or compare efficacy of different interventions in a systematic review. 
The aim of this scoping review is to identify potential treatment prescriptions and compare the outcome reporting for acupuncture clinical trials with the existing core outcome domains developed by the Harmonizing Outcome Measures for Eczema (HOME) consensus, which may help acupuncturists establish potential prioritized treatment prescriptions and choose appropriate outcomes in their future research.

\section{Methods}

2.1. Search Strategy. We conducted a systematic electronic search from Medline, PubMed, Cochrane Library, CBM, CNKI, Wanfang Database, and VIP Database from their inception to September 30, 2020. The search terms included acupuncture, acupoints, electroacupuncture, needle warming therapy, autologous whole blood injection, autohemotherapy, acupoint injection, catgut implantation, filiform steel needle, fire needle, plum-blossom needle, atopic dermatitis, chronic eczema, and atopic eczema. Language was restricted to Chinese and English. The search strategy is shown in the supplementary materials (available here).

2.2. Inclusion Criteria and Exclusion Criteria. The inclusion criteria are as follows:

(1) Population: patients diagnosed with either AD, AE, or $\mathrm{CE}$ were included as participants in this study $[2,8,9]$. There were no restrictions on age, sex, race, or region.

(2) Intervention: the included studies examined the use of acutherapy (acupuncture therapy). The studies could use multimodal interventions, but acutherapy had to be included.

(3) Comparison: comparison of the intervention group with the control group in addition to treatment methods other than acutherapy, such as drugs or other forms of traditional Chinese medicine.

(4) Outcomes: no restriction.

(5) Study types: all kinds of clinical studies including randomized clinical trials, observational studies, and case series were included in this scoping review.

The exclusion criteria are as follows:

Comments, animal experiments, and narrative reviews were excluded.

2.3. Literature Identification. We imported the results from different databases into NoteExpress 3.2.0 [10] and then deleted duplicate studies. Two reviewers (Zeng Y. J. and Zhang J. L.) conducted data identification independently. They reviewed the abstracts and full texts and extracted relevant information from the included studies. If the studies involved in comparison of therapeutic effects of different acupoint prescriptions, then the most effective acupoint prescription according to the criteria of therapeutic effect was extracted. Any discrepancies were resolved by consensus.

2.4. Data Extraction. The following information was extracted pertaining to the reference title, first author's name, publication year, study location, study design, intervention and comparisons, duration, results, and outcome measurements. These data were charted in a custom-made data extraction file and double checked by $\mathrm{ZZ}$.

2.5. Data Analysis. To overcome some weaknesses of previous studies and to determine whether acupuncture requires a different outcome set that will reflect particular effects of acupuncture on eczema symptom management, the outcomes reporting was analyzed by description analysis. Two researchers (ZZ and DZ) merged the overlapping outcomes according to the definition of core outcome domains recommended by HOME consensus including signs, symptoms, quality of life, and long-term control and grouped individual outcomes into the appropriate outcome domain independently. In addition, immune system outcomes and adverse events were added to outcome domains. These outcome sets are often preferred in what to consider as evidence relative to efficacy and safety in clinical trials of acupuncture on eczema.

Treatment prescriptions were analyzed by SPSS Modeler 18.0 [11]. We first analyzed the frequency of acupoints and meridians with text mining. Then, we determine common acupoint combinations, the support degree of different acupoint combinations, and the core acupoint association network of acupuncture for eczema by association rule mining. We obtain the core acupoints using hierarchical cluster analysis. We also analyzed the co-occurrence matrix of the top 18 acupoints using Heml 1.0.3.7 [12]. The results are illustrated by a heat map.

\section{Results}

3.1. Study Identification. A total of 1332 records were identified from the literature search, and a total of 116 eligible studies were included. The flowchart of the review is shown in Figure 1.

3.2. Characteristics of the Inclusive Studies. The characteristics of the inclusive studies are shown in Supplementary Table 1. The studies included were published between 1998 and 2020 . Most of the studies $(81 \%, 94 / 116)$ were published in 2010 and after. The majority of the studies were conducted in China $(n=111)$. The rest of the studies originated from Germany, United States, and Korea. Most of the studies used a randomized controlled trial design with two or three arms $(n=91)$. Nine studies used a control design with simple randomized assignment of participants to the experimental and control groups. 14 studies used a single group design such as a longitudinal prospective design. The duration of treatment sessions ranged from $7 \mathrm{~d}$ to $140 \mathrm{~d}$ throughout the studies, while two studies did not report duration. The 


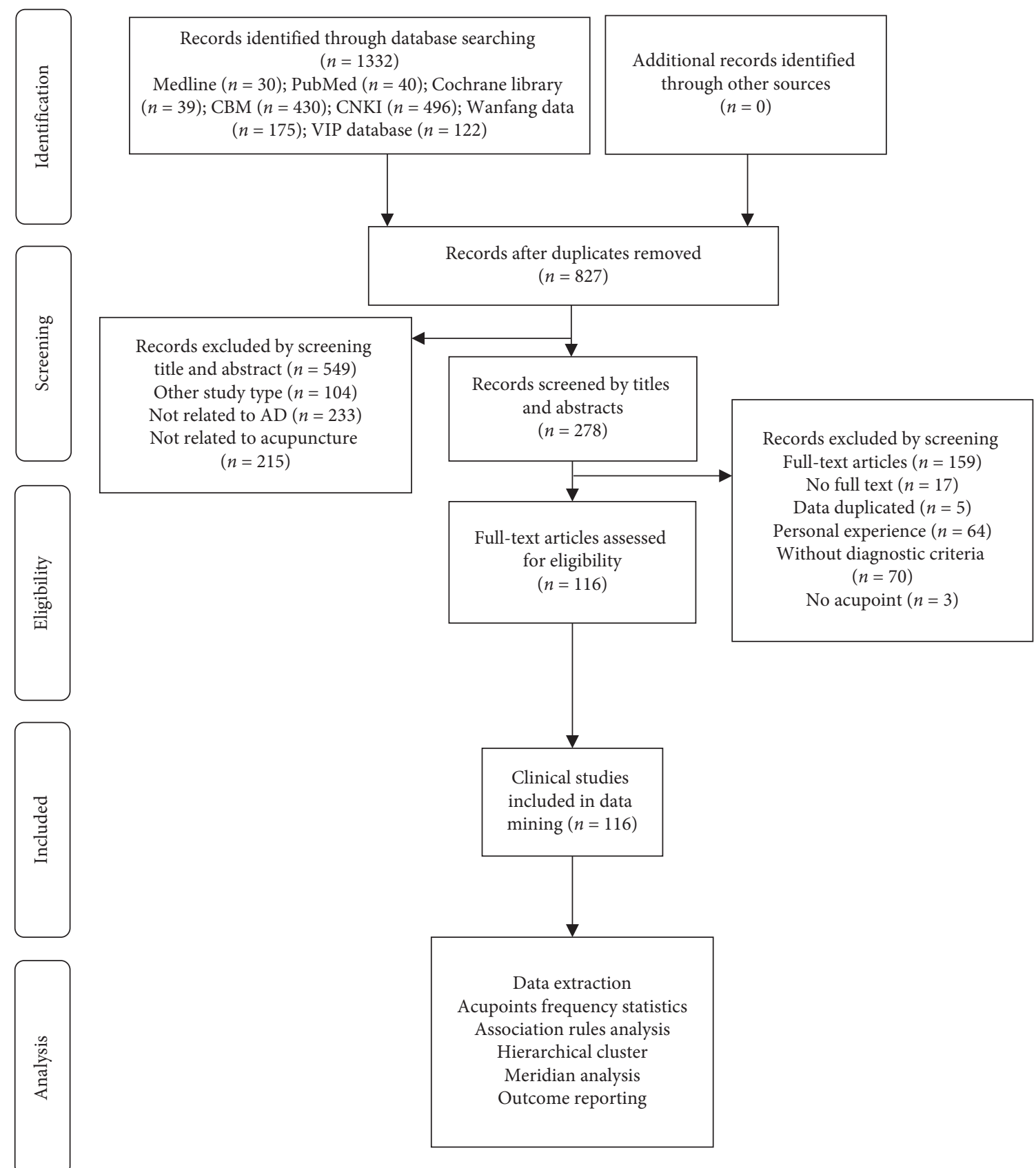

Figure 1: Flow chart of the research process.

sample sizes of all the studies range from 10 to 323 , with an average of 82 participants. Intervention procedures varied among the 116 studies. Most of the studies (44\%) included investigated manual acupuncture. Only two studies discussed electroacupuncture. Other studies involved the assessment of acupoint therapies such as autologous whole blood injection $(n=21)$, acupoint injection $(n=18)$, catgut implantation $(n=7)$, fire needle $(n=24)$, and plum-blossom needle $(n=7)$. Non-RCT studies discussed treatments such as acupuncture, autologous whole blood injection, acupoint injection, catgut implantation, fire needle, and plum-blossom needle $(n=23)$. All of the included studies demonstrated statistically significant $(P<0.05)$ improvements in at least one targeted eczema outcome.

\subsection{Results of Data Mining}

3.3.1. Frequency Statistics of Acupoints and Meridians. Acupoint frequency was analyzed based on the acquired 116 acupoint prescriptions, which involved 73 acupoints. The 
TABLE 1: Common acupoint group of acupuncture for eczema.

\begin{tabular}{lcc}
\hline No. & Acupoints & Frequency \\
\hline 1 & LI11, SP10 & $58 *$ \\
2 & LI11, ST36 & 48 \\
3 & SP10, ST36 & $40 *$ \\
4 & LI11, SP10, ST36 & 37 \\
5 & SP6, LI11 & 31 \\
6 & SP6, SP10 & 29 \\
7 & LI11, SP6, SP10 & 28 \\
8 & SP6, ST36 & 27 \\
9 & LI1, SP6, ST36 & 25 \\
10 & LI4, LI11 & 24 \\
11 & ST36, SP6, SP10 & 23 \\
12 & LI4, SP10 & 20 \\
13 & LI4, SP10, LI11 & 20 \\
14 & LI4, ST36 & 18 \\
15 & LI4, ST36, LI11 & 17 \\
\hline
\end{tabular}

* The frequency of two-acupoint combinations includes that of the two acupoints in three-acupoint combinations.

top 15 acupoints used frequently were LI11, SP10, ST36, Ashi, SP6, LI4, SP9, BL20, BL13, BL17, GV14, CV4, ST25, PC6, and BL18, in turn. There were 5 acupoints with frequency $\geq 30$, which were Ashi, LI11, SP10, ST36, and SP6. A total of 12 meridians, two extra meridians, and one Ashi point were used in acupuncture for eczema. There are 14 meridians recorded in the 116 acupoint prescription. The top 5 meridians included the Spleen Meridian of Foot-Taiyin (SP), Large Intestine Meridian of Hand-Yangming (LI), Stomach Meridian of Foot-Yangming (ST), Bladder Meridian of Foot-Taiyang (BL), and conception vessel (CV). The most frequently used meridian was SP, which was used 127 times and involved 5 acupoints. Detailed information can be checked in Figure 2.

3.3.2. Common Acupoint Combinations. A total of 15 common acupoint combinations were frequently used over 17 times. There were four acupoint combinations with frequency $\geq 35$ : LI11 and SP10, LI11 and ST36, SP10 and ST36, and LI11, SP10, and ST36. The most frequently used acupoint combination was LI11 and SP10, with 58 times. LI1 1 and ST36 was used 48 times, SP10 and ST36 was used 40 times, and LI11, SP10, and ST36 was used 37 times. Detailed information can be checked in Table 1. The cooccurrence matrix of the 18 acupoints is presented in Figure 3, which were consistent with the common acupoint combinations.

3.3.3. Association Rules of Acupoints. There were $13 \mathrm{acu}-$ point combinations with confidence levels ranging more than $80 \%$ and support degree more than $20 \%$, of which SP6, SP10 $\longrightarrow$ LI11 was listed as the association rules of $96.55 \%$, indicating that when SP6, SP10 was selected, the probability of selecting LI11 was $96.55 \%$. Also, the highest support degree was SP10 $\longrightarrow$ LI11, which was $54.31 \%$. The association rules for acupoints are described in Table 2. The core acupoint association network of acupuncture for eczema is listed in Figure 4. The degree of support from strong to weak was acupoint LI11 combined with SP10, ST36 with LI11, SP6 with LI11, SP6 with SP10, and LI4 with LI11.

3.4. Outcomes Reporting in Clinical Trials of Acupuncture for Eczema. For included clinical trials, a total of 6 outcome distinct domains were identified in the 32 outcome measurements (Table 3$)$. Seven $(21.9 \%, 7 / 32)$ outcomes were reported only once. The most frequently reported outcome was the eczema area, which was reported 97 times (83.6\%, 97/116). Immune system outcomes were assessed in 15 outcome measurements, which totally reported 37 times. Adverse events were reported 51 times. TCM syndrome, which could reflect the characteristics of TCM, was reported 4 times.

29 outcomes $(90.6 \%, 29 / 32)$ were provided definitions or OMIs. $17(53.1 \%, 17 / 32)$ outcomes were provided one OMI or definition, $4(12.5 \%, 4 / 32)$ outcomes were provided two OMIs or definitions, and $8(25.0 \%, 8 / 32)$ outcomes were provided more than two OMIs or definitions. In addition, among these outcomes, the outcome measurement times ranged from 0 to 34 , and the median time was 6.5 . Itch and eczema area had more measurement times than other outcomes did.

\section{Discussion}

The meridians, acupoint combinations, and core acupoints identified from the studies included in this scoping review broadly cover the characteristics of acupuncture prescriptions that could be offered in eczema treatment providing antipruritus and anti-inflammatory effect. Acutherapy offers an area for further clinical practice and research for treating eczema.

From the previous systematic review, we found that the majority of clinical trials of acupuncture for eczema are in low methodological quality and quality of evidence [13-15], so it is difficult to draw a definite conclusion. In addition, the researchers and clinicians may use different acupoints, so it is impossible for some clinical trials to include in systematic review when there is heterogeneity in prescription. From the results of data mining, the core acupoints may provide potential prescription to researchers.

This scoping review suggests that specific outcome measures for future clinical trials of acupuncture are clinician-reported signs, patient-reported symptoms, health-related quality of life and long-term control, TCM syndrome, immune system outcomes, and adverse events, which are different form the HOME. In addition, researchers choose different outcome measurement instruments, so some clinical trials will be excluded from systematic reviews, which produce waste and lower the value of research. For acupuncture clinical trials, following the HOME core outcome set for eczema may help improve the consistency of outcomes and outcome measurement instruments.

The theory of TCM is totally different from that of western medicine. When clinicians use acupuncture, the 


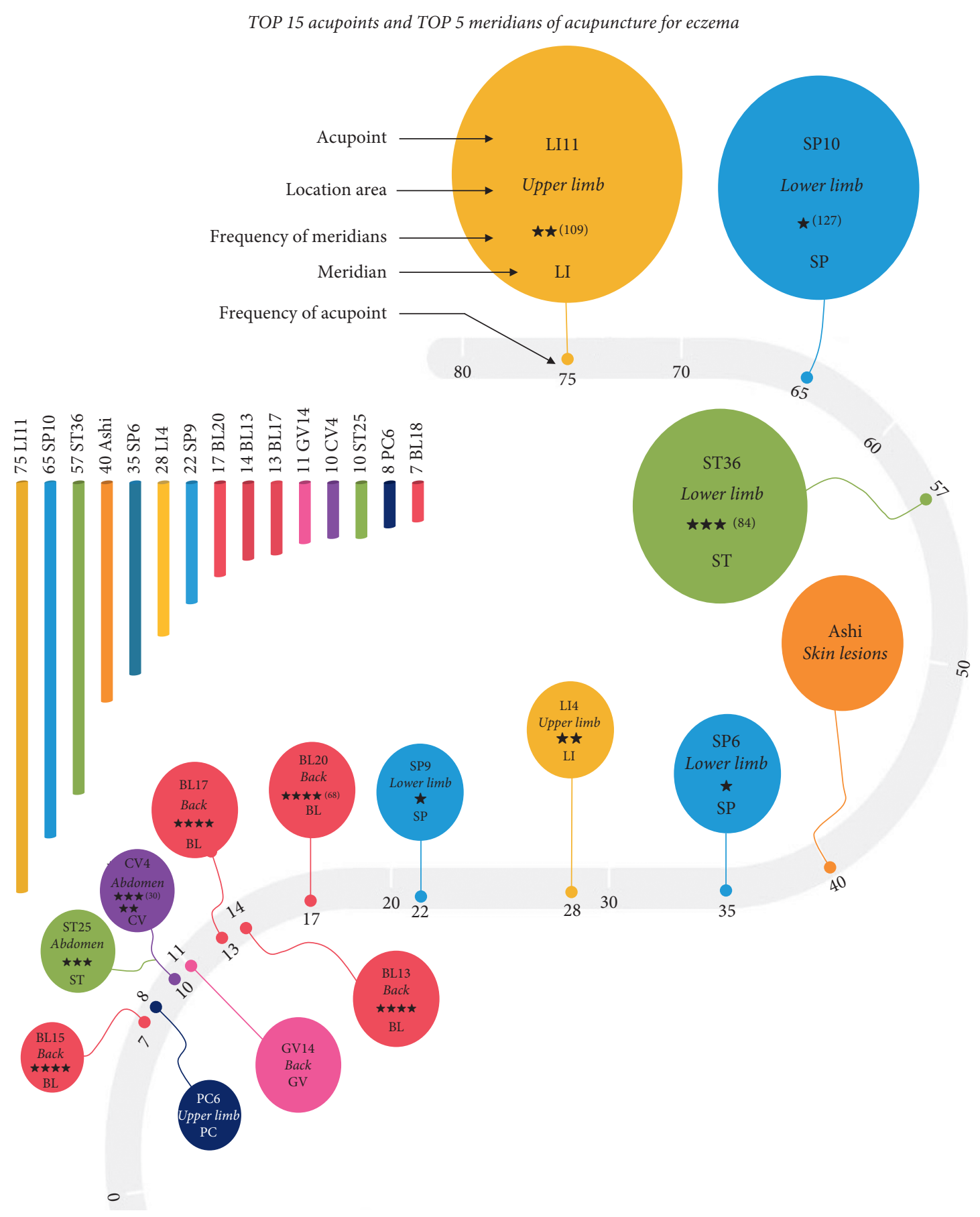

FIgURE 2: The top 15 acupoints and top 5 meridians used in acupuncture for eczema.

TCM syndromes are important factors that should be considered in the process of treatment and efficacy evaluation. Therefore, in the core outcome set for eczema, TCM syndromes should be considered. In addition, the HOME is developed by stakeholders from developed countries, and the perspectives of professionals and patients from low- and middle-income countries are missed. Therefore, the core outcome set for eczema should be achieved consensus in researchers, clinicians, and patients in China.
The current research had several strengths. To our knowledge, this was the first study that used bioinformatics methods and searched Chinese and English databases to assess which acupoints have been used to treat eczema. First, previous research was limited in that it could only "qualitatively" interpret the characteristics of acupoint prescriptions used in eczema treatment. We overcame this limitation by extracting significant "quantitative" characteristics using the association rule mining. Second, we explored core acupoints used in eczema treatment. Moreover, we evaluated 


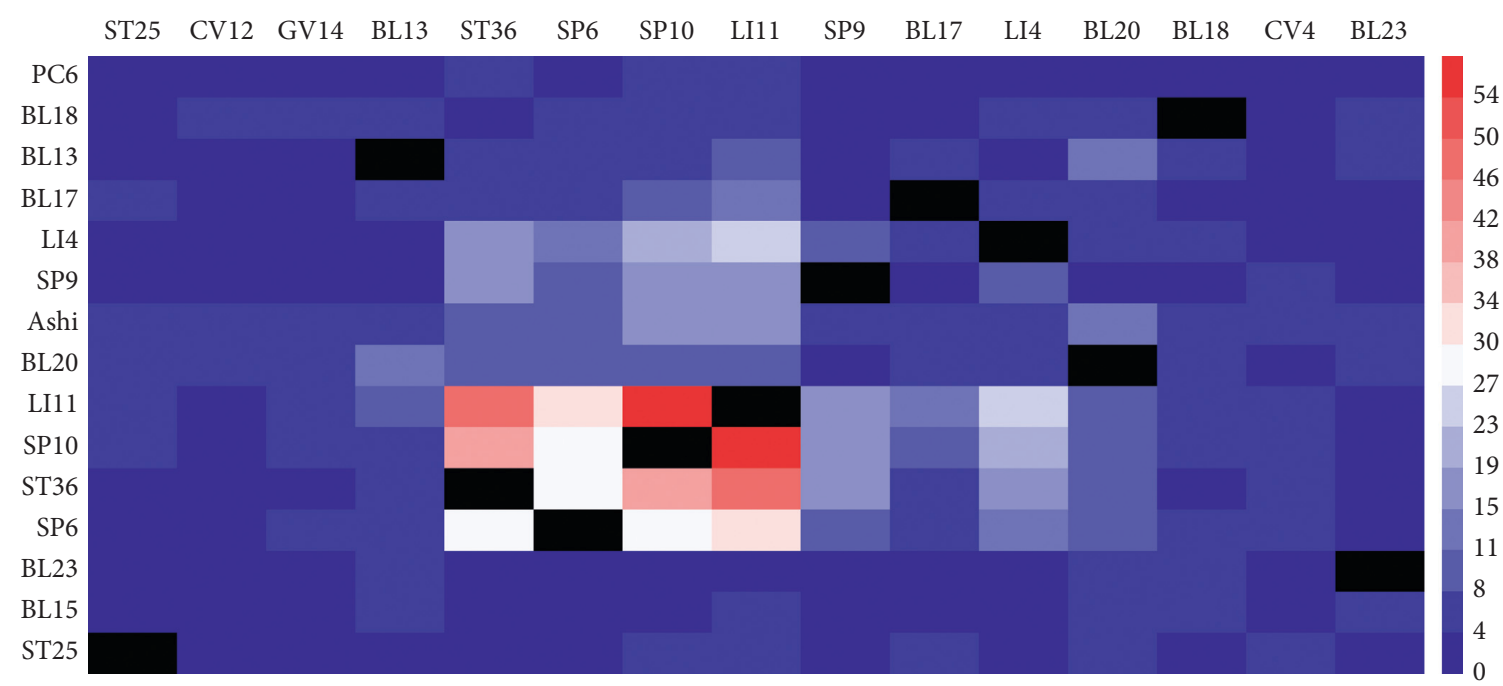

Figure 3: Co-occurrence matrix of acupoints.

TABLE 2: Association rules of acupoints.

\begin{tabular}{|c|c|c|c|}
\hline Number & Acupoints & Confidence level (\%) & Support degree (\%) \\
\hline 1 & SP6, SP10 —LI11 & 96.55 & 25.00 \\
\hline 2 & $\mathrm{SP} 6 \longrightarrow \mathrm{LI} 11$ & 93.94 & 28.45 \\
\hline 3 & SP6, ST36 $\longrightarrow$ LI11 & 92.59 & 23.28 \\
\hline 4 & ST36, SP10 $\longrightarrow$ LI11 & 92.50 & 34.48 \\
\hline 5 & $\mathrm{SP} 10 \longrightarrow \mathrm{LI} 11$ & 92.06 & 54.31 \\
\hline 6 & $\mathrm{SP} 6, \mathrm{LI} 11 \longrightarrow \mathrm{SP} 10$ & 90.32 & 26.72 \\
\hline 7 & $\mathrm{SP} 6 \longrightarrow \mathrm{SP} 10$ & 87.88 & 28.45 \\
\hline 8 & ST36 $\longrightarrow$ LI11 & 87.27 & 47.41 \\
\hline 9 & $\mathrm{LI} 4 \longrightarrow \mathrm{LI} 11$ & 85.71 & 24.14 \\
\hline 10 & $\mathrm{SP} 6, \mathrm{ST} 36 \longrightarrow \mathrm{SP} 10$ & 85.19 & 23.28 \\
\hline 11 & $\mathrm{LI} 4, \mathrm{LI} 11 \longrightarrow \mathrm{SP} 10$ & 83.33 & 20.69 \\
\hline 12 & $\mathrm{SP6} \longrightarrow \mathrm{ST} 36$ & 81.82 & 28.45 \\
\hline 13 & $\mathrm{SP6}, \mathrm{LI} 11 \longrightarrow \mathrm{ST} 36$ & 80.65 & 26.72 \\
\hline
\end{tabular}

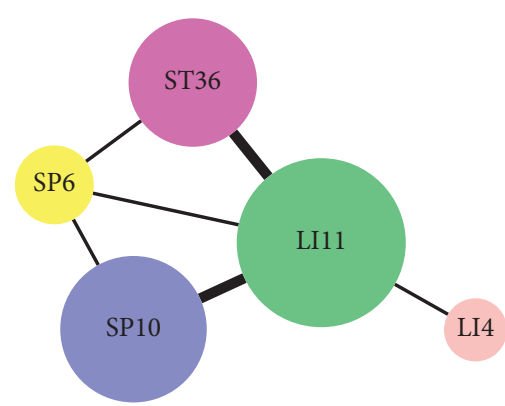

Figure 4: Core acupoint association network of acupuncture for eczema. The size of the circle represents the frequency, and the width of the line indicates the support degree.

the quality of outcome reporting, OMIs/definitions, and outcome measurement times.

\section{Limitations}

Our research had several limitations. The present study was based on the frequency of prescription used in the clinical practice and literature. For this reason, we could not evaluate new candidate acupoints emerging from recent experimental studies. Relatedly, we did not evaluate the clinical effectiveness of each prescription and adjunct points prescribed based on syndrome differentiation in our study. Finally, the acupoint combinations and core acupoints of acupuncture for eczema were rarely confirmed by large-scale multicenter clinical trials 
TABLE 3: The outcomes reporting in clinical trials of acupuncture for eczema.

\begin{tabular}{|c|c|c|c|}
\hline Domains/outcomes & $\begin{array}{l}\text { Outcomes reporting } \\
(\mathrm{n})\end{array}$ & OMIs/definitions & $\begin{array}{l}\text { Measurement time point } \\
(n)\end{array}$ \\
\hline \multicolumn{4}{|l|}{ Clinical signs } \\
\hline Erythema & 65 & $\begin{array}{l}\text { SCORAD, EASI, Likert scale, Chinese self-made } \\
\text { questionnaire }\end{array}$ & 30 \\
\hline Oozing/crust & 21 & SCORAD, EASI, Likert scale, POME & 18 \\
\hline $\begin{array}{l}\text { Induration/edema/ } \\
\text { papulation }\end{array}$ & 64 & $\begin{array}{l}\text { SCORAD, EASI, Likert scale, Chinese self-made } \\
\text { questionnaire }\end{array}$ & 31 \\
\hline Excoriation & 59 & $\begin{array}{c}\text { SCORAD, EASI, Likert scale, POME, Chinese self-made } \\
\text { questionnaire }\end{array}$ & 29 \\
\hline Lichenification & 60 & SCORAD, EASI, Chinese self-made questionnaire & 28 \\
\hline Dryness & 9 & SCORAD, POME & 13 \\
\hline Lesion area & 97 & $\begin{array}{l}\text { SCORAD, EASI, Likert scale, Chinese self-made } \\
\text { questionnaire }\end{array}$ & 32 \\
\hline Skin temperature & 2 & Infrared radiation thermometer & 3 \\
\hline \multicolumn{4}{|l|}{ Symptoms } \\
\hline Itch & 91 & VAS, EIQ, POME, Chinese self-made questionnaire & 34 \\
\hline Bleeding & 1 & POME & 3 \\
\hline Cracks & 1 & Chinese self-made questionnaire & 2 \\
\hline Sleep loss & 10 & VAS, ISI, PSQI, SRSS, Likert scale & 13 \\
\hline TCM symptoms & 4 & Chinese self-made questionnaire & 8 \\
\hline \multicolumn{4}{|c|}{ Heath-related quality of life } \\
\hline \multicolumn{4}{|c|}{ Long-term control of flares } \\
\hline Recurrence rate & 20 & - & 28 \\
\hline \multicolumn{4}{|l|}{ Immune system outcomes } \\
\hline BAT & 1 & FLOW-CAST basophil activation test & 3 \\
\hline BASO & 1 & Blood routine examination & 2 \\
\hline EOS & 3 & $\begin{array}{c}\text { Automated hematology analyzer, blood routine } \\
\text { examination }\end{array}$ & 5 \\
\hline $\mathrm{CD} 3+$ & 2 & Flow cytometry & 3 \\
\hline $\mathrm{CD} 4+$ & 4 & Flow cytometry & 11 \\
\hline $\mathrm{CD} 8+$ & 4 & Flow cytometry & 11 \\
\hline IFN- $\gamma$ & 3 & ELISA & 4 \\
\hline TNF- $\alpha$ & 2 & ELISA & 3 \\
\hline IL-2 & 3 & ELISA & 4 \\
\hline IL-4 & 5 & ELISA & 5 \\
\hline IL-5 & 3 & ELISA & 4 \\
\hline IL-12 & 1 & ELISA & 2 \\
\hline IL-18 & 1 & ELISA & 2 \\
\hline $\operatorname{IgE}$ & 5 & Immunoturbidimetry assay, ELISA & 11 \\
\hline CRP & 1 & ELISA & 3 \\
\hline \multicolumn{4}{|l|}{ Others } \\
\hline \multirow{2}{*}{$\begin{array}{l}\text { Adherence/compliance } \\
\text { Adverse events/side } \\
\text { effects }\end{array}$} & 20 & - & 0 \\
\hline & 51 & - & 20 \\
\hline
\end{tabular}

BAT: basophil activation test; EOS: eosinophil count; BASO: basophil; CRP: C-reactive protein; EASI: Eczema Area and Severity Index; POEM: Patient-Oriented Eczema Measure; EIQ: Eppendorf Itch Questionnaire; VAS: Visual Analogue Scale; SCORAD: Scoring Atopic Dermatitis Index; DLQI: Dermatology Life Quality Index; EPQOLS: quality of life scale for chronic eczema; ISI: Insomnia Severity Index; PSQI: Pittsburgh Sleep Quality Index; SRSS: Sleep Self-Rating Scale.

or animal experiments. Further clinical/experimental studies are needed to assess whether the results derived from our research have real meaning for applications.

\section{Conclusions}

The preference of core acupoints is LI11, SP10, ST36, SP6, and LI4 in acupuncture clinical studies on eczema. In the future, the researchers could focus on them to prove the efficacy of potential core acupoints. However, researchers should use the core outcome set for eczema to improve the consistency of outcomes and outcome measurement instruments so that the clinical trials will be not exclude from systematic reviews because of the heterogeneity of outcome reporting and outcome measurement instruments.

\section{Abbreviations}

AE: $\quad$ Atopic eczema

$\mathrm{AD}$ Atopic dermatitis 
AMED: Japan Agency for Medical Research and Development

ARM: $\quad$ Association rule mining

BASO: Basophil

BAT: Basophil activation test

BL: $\quad$ Bladder Meridian of Foot-Taiyang

CBM: $\quad$ Chinese biomedical medicine

CCT: Clinical control trial

CE: $\quad$ Chronic eczema

CMCC: Chinese medical current content

CNKI: China National Knowledge Infrastructure

CRP: C-reactive protein

DLQI: Dermatology Life Quality Index

EA: $\quad$ Electroacupuncture

EASI: Eczema Area and Severity Index

EIQ: $\quad$ Eppendorf Itch Questionnaire

EOS: $\quad$ Eosinophil count

EPQOLS: Quality of life scale for chronic eczema

ERK: $\quad$ Extracellular regulated protein kinases

GB: Gallbladder Meridian of Foot-Shaoyang

GV: $\quad$ Governor vessel

IFN: Interferon

Ig: Immunoglobulin

IL: Interleukin

ISI: Insomnia Severity Index

LI: $\quad$ Large Intestine Meridian of Hand-Yangming

LR: $\quad$ Liver Meridian of Foot-Jueyin

POEM: Patient-oriented eczema measure

PSQI: $\quad$ Pittsburgh Sleep Quality Index

RCT: $\quad$ Randomized control trial

SCORAD: Scoring Atopic Sermatitis Index

SP: $\quad$ Spleen Meridian of Foot-Taiyin

SRSS: $\quad$ Sleep Self-Rating Scale

ST: $\quad$ Stomach Meridian of Foot-Yangming

TNF: $\quad$ Tissue necrosis factor

VAS: $\quad$ Visual Analogue Scale.

\section{Data Availability}

The datasets used and/or analyzed during the current study are available from the corresponding author upon reasonable request.

\section{Conflicts of Interest}

The authors declare that they have no conflicts of interest.

\section{Authors' Contributions}

ZZ and ML drafted the manuscript. YZ, JZ, and YL extracted data from articles. $\mathrm{ZZ}$ and $\mathrm{YZ}$ contributed to the data extracting and assessment. DZ, RQ, and HS revised the manuscript. All authors read and approved the final manuscript.

\section{Acknowledgments}

This work was supported by the National Natural Science Foundation of China (grant no. 81774390) and the
Traditional Chinese Medicine Bureau of Guangdong Provincial (grant nos. 20201022 and 20211258). All funding sources were used for data collection.

\section{Supplementary Materials}

The detailed search strategy and characteristics of the inclusive studies are shown in Supplementary materials. . (Supplementary Materials)

\section{References}

[1] B. Cabanillas, A. C. Brehler, and N. Novak, "Atopic dermatitis phenotypes and the need for personalized medicine," Current Opinion in Allergy and Clinical Immunology, vol. 17, no. 4, pp. 309-315, 2017.

[2] S. Weidinger and N. Novak, "Atopic dermatitis," Lancet, vol. 387, no. 10023, pp. 1109-1122, 2016.

[3] B. Zhao, China Clinical Dermatology, Phoenix science press, Nanjing, China, 2010.

[4] S. G. Johansson, T. Bieber, R. Dahl et al., "Revised nomenclature for allergy for global use: report of the nomenclature review committee of the World allergy organization," Journal of Allergy and Clinical Immunology, vol. 113, no. 5, pp. 832836, 2004.

[5] E. Ernst, "The usage of complementary therapies by dermatological patients: a systematic review," British Journal of Dermatology, vol. 142, no. 5, pp. 857-861, 2000.

[6] B. L. Vieira, N. R. Lim, M. E. Lohman et al., "Complementary and alternative medicine for atopic dermatitis: an evidencebased review," American Journal of Clinical Dermatology, vol. 17, no. 6, pp. 557-581, 2016.

[7] S. Kang, Y. K. Kim, M. Yeom et al., "Acupuncture improves symptoms in patients with mild-to-moderate atopic dermatitis: a randomized, sham-controlled preliminary trial," Complementary Therapies in Medicine, vol. 41, pp. 90-98, 2018.

[8] X. Yao, Z.-Q. Song, and W. Li, "Guidelines for the diagnosis and treatment of atopic dermatitis in China," Chinese Journal of Dermatology, vol. 4, no. 2, pp. 81-88, 2020.

[9] J. M. Hanifin and G. Rajka, "Diagnostic features of atopic dermatitis," Acta Dermato-Venereologica, vol. 9, no. 2, pp. 44-47, 1980.

[10] NoteExpress, 2020, http://www.inoteexpress.com/aegean/.

[11] I. H. Witten, E. Frank, M. A. Hall et al., Data Mining: Practical Machine Learning Tools and Techniques, Morgan Kaufmann, Burlington, MA, USA, 2nd edition, 2016.

[12] Heml, Heatmap Illustrator, 2020, http://hemi.biocuckoo.org/ down.php.

[13] Z. B. Yang and W. J. Zhang, "Systematic assessment of acupuncture treatment on eczema," International Journal of Chinese Medicine, vol. 32, no. 3, pp. 250-251, 2010.

[14] H. Y. Tan, G. B. Lenon, A. L. Zhang et al., "Efficacy of acupuncture in the management of atopic dermatitis: a systematic review," Clinical and Experimental Dermatology, vol. 40, no. 7, pp. 711-715, 2015.

[15] R. Jiao, Z. Yang, Y. Wang et al., "The effectiveness and safety of acupuncture for patients with atopic eczema: a systematic review and meta-analysis," Acupuncture in Medicine, vol. 38, no. 1, pp. 3-14, 2020. 